- The extraction of teeth for orthodontic purposes has always been a controversial area. It is not possible to treat all malocclusions without taking out teeth

- Where extractions are indicated, first premolars are most commonly extracted but there are reasons for extracting elsewhere in the arch and this will involve other teeth

- The use of fixed appliances has considerably changed extraction viewpoints

\title{
Orthodontics. Part 8: Extractions in orthodontics
}

\author{
H. Travess ${ }^{1}$, D. Roberts-Harry ${ }^{2}$ and J. Sandy ${ }^{3}$
}

\section{NOW AVAILABLE AS A BDJ BOOK}

\author{
Extractions in orthodontics remains a relatively controversial area. It is not possible to treat all \\ malocclusions without taking out any teeth. The factors which affect the decision to extract \\ include the patient's medical history, the attitude to treatment, oral hygiene, caries rates and \\ the quality of teeth. Extractions of specific teeth are required in the various presentations of \\ malocclusion. In some situations careful timing of extractions may result in spontaneous \\ correction of the malocclusion.
}

ORTHODONTICS

1. Who needs orthodontics?

2. Patient assessment and examination I

3. Patient assessment and examination II

4. Treatment planning

5. Appliance choices

6. Risks in orthodontic treatment

7. Fact and fantasy in orthodontics

8. Extractions in orthodontics

9. Anchorage control and distal movement

10. Impacted teeth

11. Orthodontic tooth movement

12. Combined orthodontic treatment

Senior Specialist Registrar in Orthodontics; ${ }^{3}$ Professor of Orthodontics, Division of Child Dental Health, University of Bristol Dental School, Lower Maudlin Street, Bristol BS1 2LY; ${ }^{2 *}$ Consultant Orthodontist, Orthodontic Department, Leeds Dental Institute, Clarendon Way, Leeds LS2 $9 \mathrm{LU}$ ${ }^{*}$ Correspondence to: D. Roberts-Harry E-mail: robertsharry@btinternet.com

\section{Refereed Paper}

doi:10.1038/sj.bdj.4810979

๑ British Dental Journal 2004; 196:

195-203
The role of extractions in orthodontic treatment has been a controversial subject for over a century. It is fair to say that even today, opinion is divided on whether extractions are used too frequently in the correction of malocclusion.

Angle ${ }^{1}$ believed that all 32 teeth could be accommodated in the jaws, in an ideal occlusion with the first molars in a Class I occlusion, ie with the mesiobuccal cusp of the upper first molar occluding in the buccal groove of the lower first molar. Extraction was anathema to his ideals, as he believed bone would form around the teeth in their new position, according to Wolff's law. ${ }^{2}$ This was criticised in 1911 by Case who believed extractions were necessary in order to relieve crowding and aid stability of treatment. $^{3}$

Two of Angle's students at around the same time but in different countries considered the need for extractions in achieving stable results. Tweed became disappointed in the results he was achieving and decided to re-treat a number of patients who had suffered relapse following orthodontic treatment (at no further cost) using extraction of four premolar units. ${ }^{4}$

The demonstration of his results to the profession in America resulted in a change of philosophy in the 1940s to extraction-based techniques. Begg, in Australia, studied Aboriginal skulls and noted a large amount of occlusal and more importantly interproximal wear. ${ }^{5}$ He argued that premolar extractions were required in order to compensate for the lack of interproximal wear seen in the modern Australian dentition, through lack of a coarse diet. He also developed a technique that relied on extractions to create much of the anchorage needed for treatment.
Recently, the extraction debate has reopened, with some individuals believing that expansion of the jaws and retraining of posture can obviate the need for extractions and produce stable results. These claims are for the most part unsubstantiated. If teeth are genuinely crowded as opposed to being irregular then arch alignment can be achieved by one of the following:

- Enlargement of the archform

- Reduction in tooth size

- Reduction in tooth number

Arch expansion can be achieved by moving teeth buccally and labially (ie lateral and anterio posterior expansion) but the long-term stability and whether bone grows as teeth are moved through cortical plates remain contentious issues. In the maxilla there is a suture which remains patent in some patients into the second decade. This can also be used in expansion in that it can be 'split' with rapid maxillary expansion. The split suture fills in with bone and thus a wider arch to accommodate teeth is created. There is no good evidence that this method of expansion produces a more stable result than any other method. Longitudinal studies provide useful guidance on whether arch expansion produces stability. These are difficult studies to conduct but increasing mandibular length to accommodate teeth relapses in nearly 90\% of cases with resulting unsatisfactory anterior tooth alignment. ${ }^{6}$

Reduction in tooth size, particularly in the labial segments with interdental stripping, is another potential mechanism to relieve crowding. Variable relapse has been reported but one study noted relapse of some degree in all cases. ${ }^{7}$ 
This work was done over 25 years ago and does not reflect contemporary use of inter-dental enamel reduction or current retention regimes.

The reduction in tooth number is usually achieved with extractions and these cases ideally need to be compared with treated non extraction cases with spacing, cases treated by arch expansion to accommodate crowding and untreated normal occlusions. In a review of these issues it was concluded that arch length reduces in most cases, including untreated normal occlusion. Any lateral expansion across the mandibular canines decreases after treatment but this is also seen in those cases which have no orthodontic treatment. It was further recognised that mandibular anterior crowding is a continuing phenomenon seen in patients into the fourth decade and likely beyond. ${ }^{8}$ The degree of anterior crowding seen at the end of retention is variable and unpredictable.

Proffit $^{9}$ in a 40 -year review of extraction patterns showed $30 \%$ of cases were treated with extractions in 1953, 76\% in 1968 and 28\% in 1993. He suggested the decline in extractions since 1968 was because of concern over facial profile, tempromandibular joint dysfunction (TMD) and stability; the change from the Begg appliance, largely an extraction-based technique to the straight wire technique, which seems to require fewer extractions. The latter may also result with a change in mindset and the use of headgear and prolonged retention.

A dogmatic approach is inadvisable and each case must be assessed on its merits. Some cases, especially where the crowding is mild may not need tooth removal, and a more sensible approach based on the requirements of the individual case rather that the two extremes seen in the past century is advised. Interestingly, in a follow up study over a 15 year period in

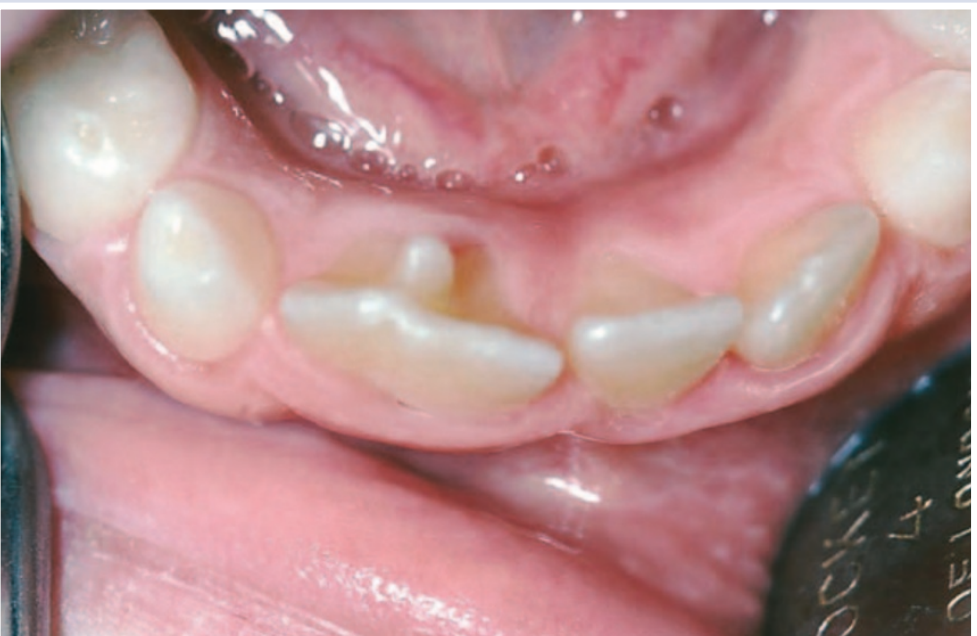

Fig. 1 Illustration of a macrodont tooth in the lower labial segment, which also exhibits a talon cusp. Alignment and arch co-ordination is hindered by the size of the tooth and the talon cusp. Some enamel reduction can be undertaken to reduce the width of the tooth but care must be taken not to breach the enamel. In the upper arch, reduction of a talon cusp can help correct an increased overjet, although radiographic examination of pulp chambers in the talon cusp is essential
Scotland, orthodontics replaced caries as the commonest reason for extraction in patients under 20 years of age. ${ }^{10}$ All extractions are traumatic as far as the patient is concerned and clinicians will seek non-extraction solutions where possible. In the late mixed dentition, between $3 \mathrm{~mm}$ and $4 \mathrm{~mm}$ of space can be preserved in the lower arch by simply fitting a lingual arch. If this is coupled with molar and premolar expansion of just $2 \mathrm{~mm}$ (with no lower canine exapansion) and interdental enamel reduction between anterior contact points then a large proportion of otherwise 'crowded' cases can be treated without the loss of permanent teeth. The decision on whether or not to extract teeth is based on an assessment of many factors including crowding, increase in overjet, change in arch width, curve of Spee, anchorage requirements and other more esoteric factor such as adjusting the torque of the anterior teeth. It is also worth mentioning that the concept of space analysis is probably underused in the United Kingdom, but this is routinely applied elsewhere. This analysis enables a rationale and methodical approach to treatment planning before extractions are recommended. ${ }^{11}$ It is important then to realise that there are a variety of options as far as mild to moderate crowding cases are concerned.

\section{FACTORS AFFECTING THE DECISION TO EXTRACT}

It is important to consider the patient as a whole in treatment planning. Medical history, attitude to treatment, oral hygiene, caries rate and the quality of the teeth are important. Patients with cardiac anomalies are at risk of complications during orthodontic treatment and consultation with a cardiologist is important. If necessary, extractions should be covered with appropriate antibiotics and impacted teeth may be best removed rather than aligned as traction to unerupted teeth may pose an increased risk to these patients. ${ }^{12}$

The quality and prognosis of the teeth should be carefully considered, as this may override other factors. Hypoplastic, heavily restored or carious teeth should generally be removed in preference to healthy teeth. This is especially true in the labial segments where aesthetics are difficult to maintain with loss of an incisor or canine.

Teeth of abnormal form or size may be considered for removal as they can look unsightly and be difficult to align. For example, a dens-indente may compromise the long-term prognosis of a tooth, or a talon cusp may hinder arch co-ordination during treatment. Dilacerated teeth should be carefully assessed to see if crown alignment is achievable. Often extraction of these teeth is the only option. Macrodont teeth, geminated or fused, need careful consideration (Fig. 1). The aesthetics are often poor but extraction can result in an excess amount of space, which may prolong orthodontic treatment. Where supplemental teeth are present, extraction may result in spontaneous correction of any crowding (Fig. 2). 


\section{EASE OF EXTRACTION, AND THE PRESENCE OF IMPACTED TEETH}

The extraction of teeth is a potentially traumatic experience. The decision to extract should be made with an awareness of the risks of treatment, including the psychological impact of the procedure. The General Dental Council in its guidance to dentists of professional and personal conduct makes it clear that dentists who refer patients for general anaesthesia must make it clear what justification there is for the procedure. The duties of the treating dentist include a thorough and clear explanation of the risks involved as well as the alternative methods of pain control available. The use of general anaesthesia is usually considered in dealing with unerupted teeth, first molars, multiple extractions in four quadrants and specific phobias.

If teeth are impacted or ectopically positioned, extraction of an erupted tooth can guide the path of eruption of the impacted tooth and obviate the need for minor oral surgery. For example, the impaction of a lower second premolar may be relieved by the removal of the first premolar or first molar, which only requires local analgesia and is less traumatic than the removal of the impacted tooth (Fig. 3). In Figure 4, eruption of the upper second premolars resulted in severe resorption of the roots of the upper first molars. Extracting these molars would be fairly atraumatic and allow the second premolars to erupt into the mouth. Similarly, if unerupted permanent canines are palatally positioned judicious removal of the deciduous canines can improve the path of eruption of the permanent teeth and may help to avoid lengthy orthodontic treatment. ${ }^{13}$

\section{CORRECTION OF OVERBITE}

Space closure with fixed appliances tends to increase the overbite and therefore extractions in the lower arch in deep bite cases should be undertaken with caution. In some malocclusions, where the anterior face height is reduced, extractions can make space closure difficult and great care must be taken in diagnosis before this decision is made. It is important to recognise whether a case is genuinely crowded or whether the teeth are displaced lingually as in a Class II Division 2 case. Lingually displaced lower labial segments are frequently not crowded, even though they may appear to be so.

Proclination of the lower labial segment also reduces the overbite, as well as overjet, and may obviate the need for extractions. However, this treatment approach should be undertaken cautiously as uncontrolled and excessive proclination of the lower incisors can be unstable and should only be undertaken in selected cases by experienced clinicians. Flattening of an accentuated curve of Spee in order to reduce an overbite, where proclination is contraindicated, does require space, for which the extraction of lower teeth can sometimes be considered. The space required to flatten a curve of Spee has historically been over rated, the amount of space required is a

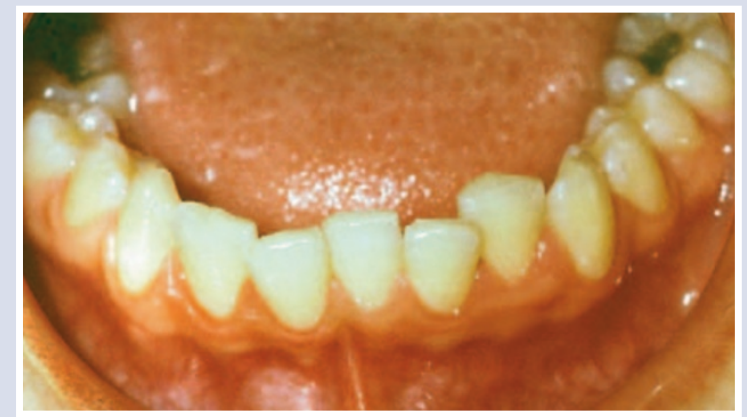

b

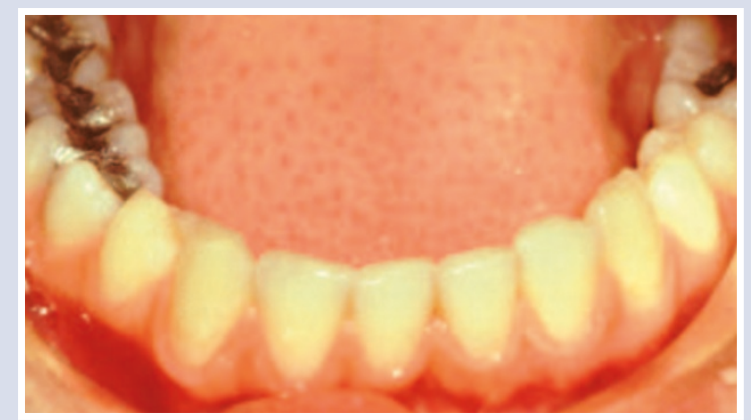

Fig. 2 A supplemental lower incisor (a) was removed, resulting in spontaneous correction of crowding in the lower labial segment (b)
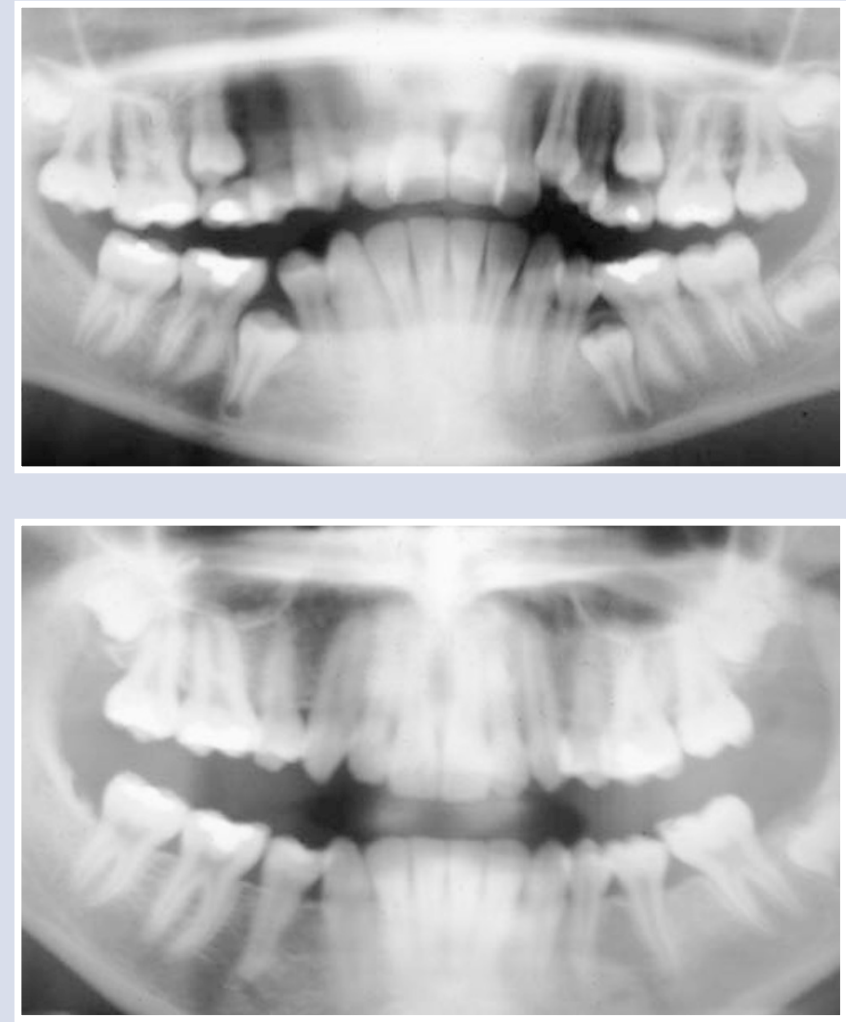

Fig. 3 This case presented with missing upper first premolars and lower right third molar, with vertically impacted lower second premolars. (a) Both lower first molars are heavily filled and would be ideal for extraction to allow eruption of the second premolars. However the missing third molar on the right resulted in extraction of the lower right first premolar and the lower left first molar. Spontaneous alignment occurred (b) with both impacted premolars erupting successfully into the occlusion with no active treatment 

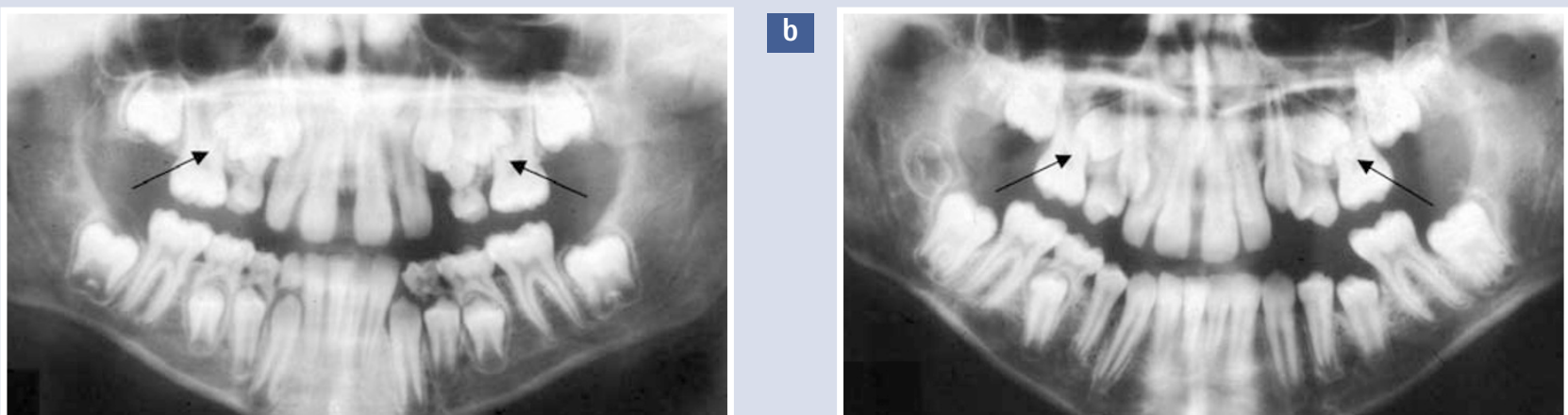

Fig. 4 In this case, the erupting upper second premolars showed some resorption of the mesial roots of the upper first molars. (a) Progressive resorption of the mesial roots of the molars was seen on subsequent radiographs (b), which progressed to such an extent (c) that both upper first molars required extraction, allowing eruption of the second premolars

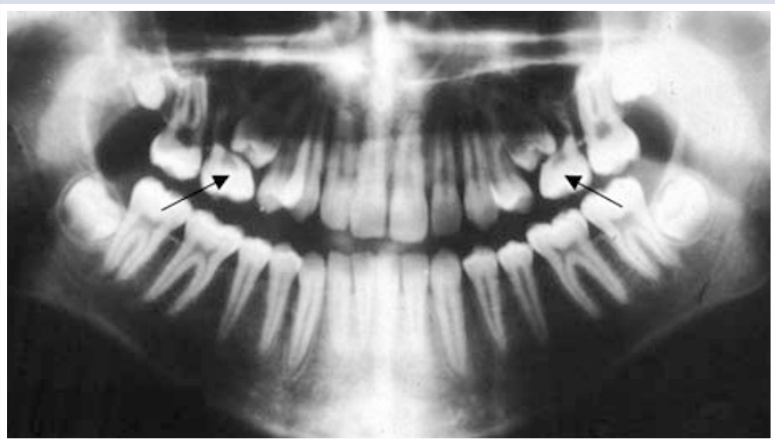

$1-2 \mathrm{~mm}$ when the curve is severe and there is no crowding. It is difficult then to justify extracting teeth purely for the sake of creating space to flatten an occlusal curve. The greatest challenge is the mechanical control of the teeth to prevent excessive proclination of the lower incisors. This usually occurs because the intrusion force is at some distance labial to the centre of resistance of the incisors and lingual crown torque is needed to prevent the labial movement of the incisors.

\section{EXTRACTION OF SPECIFIC TEETH}

Despite the factors discussed above, certain teeth are extracted preferentially for orthodontic reasons. A survey of extraction patterns in the hospital orthodontic service (Table 1) showed that first premolars were most commonly extracted (59\%) followed by second premolars (13\%). Permanent molars accounted for 19\% of extractions (12\% for first molars and 7\% for second molars). Only $1 \%$ of patients had incisor extractions. ${ }^{14}$

The high percentage of premolar extractions is related to their position in the arch and the

\begin{tabular}{lc}
$\begin{array}{l}\text { Table } 1 \text { Table of percentage extractions according to } \\
\text { tooth type }\end{array}$ \\
\hline Tooth & \% removed \\
\hline Central incisor & 1 \\
Lateral incisor & 3 \\
Canine & 4 \\
First premolar & 59 \\
Second premolar & 13 \\
First molar & 12 \\
Second molar & 7
\end{tabular}

timing of their eruption. They are often ideal for the relief of anterior and posterior crowding. However, each patient should be seen as an individual and their treatment planned according to the merits of the malocclusion,

\section{Lower incisors}

In general, removal of a lower incisor should be avoided, as the inter-canine width tends to decrease which can result in crowding developing in the upper labial segment or the overjet increasing. However, a number of situations do exist in which a lower incisor may be considered as part of an orthodontic treatment plan and fixed appliances are generally required in these cases. These include situations where a lower incisor is grossly displaced from the arch form or 'ectopic' and space is required to align the teeth. This is best considered in adults and especially those who have had previous loss of premolar units in each quadrant and present with late lower labial segment crowding (Fig. 5). Class III cases at the limit of their growth can be camouflaged with loss of a lower incisor, to allow the lower labial segment to be tipped lingually, correcting the incisor relationship. This also tends to increase the overbite, which is helpful in these cases. ${ }^{15}$ Treatment of Class I cases with moderate lower labial segment crowding of up to $5 \mathrm{~mm}$ (ie the size of a lower incisor) may be treated with loss of a lower incisor. An increase in overjet or a slightly Class III buccal segment relation may be an undesirable side effect. ${ }^{16}$ Cases where a tooth size discrepancy exists, for example with upper peg shaped laterals or missing upper lateral incisors may also benefit from the loss of a lower incisor. A Bolton analysis (a measure of tooth 
size discrepancies) may be used to analyse the extent of the disproportion. A Kesling set up ${ }^{17}$ (where the anterior teeth are sectioned from a plaster model and re-positioned in wax as a trial set up, having left out a lower incisor) may be helpful in predicting the final outcome (Fig. 6).

\section{Upper Incisors}

Upper incisors are rarely the extraction of choice to treat a malocclusion. However, the upper labial segment is particularly at risk from trauma, especially in Class II Division 1 cases with large overjets. In situations where the long-term prognosis of an incisor is poor, for example, the incisor is non vital, root filled, dilacerated or of abnormal form, the tooth should be considered for extraction as part of the orthodontic treatment plan. Full consideration should be given to the resulting occlusion and aesthetics. Placing a lateral incisor in a central incisor position rarely gives a good result because the root of the tooth is narrow and the emergence angle of the built up crown is poor. In some cases transplantation of a premolar with a developing root into the incisor socket can relieve crowding in the lower arch and provide a useful replacement in the upper labial segment (Fig. 7).

Where lateral incisors are diminutive or missing, space closure or space maintenance can be considered more equally. Attention must be paid to the shape, size, gingival height and colour of the canine if a good aesthetic result is to be achieved. In many cases the canines can be disguised as lateral incisors by selective grinding, and where appropriate, aesthetic build-ups.

\section{Canines}

These teeth are rarely considered for extraction unless very ectopic (Fig. 8). The loss of a canine makes canine guidance impossible and may compromise a good functional occlusal result. Contact between a premolar and lateral incisor is often poor and canines can act as ideal abutment teeth because of their long root length and resistance to periodontal problems. Palatally ectopic canines can sometimes be in unfavourable positions for alignment, and lower ectopic canines often require extraction rather than alignment. In many of the former cases the first premolar can be aligned with a mesial inclination and rotated mesio-palatally to hide the palatal cusp and provide a better aesthetic result.

\section{Premolars}

Premolars are often ideal for the relief of both anterior and posterior crowding, the first and second premolars have similar crown forms, which means that an acceptable contact point can be achieved between the remaining premolar and the adjacent molar and canine. The choice between first or second premolar depends on a number of factors: for example, the degree of crowding, the anchorage requirements, the overjet and overbite.

In Class I cases where crowding exists and the
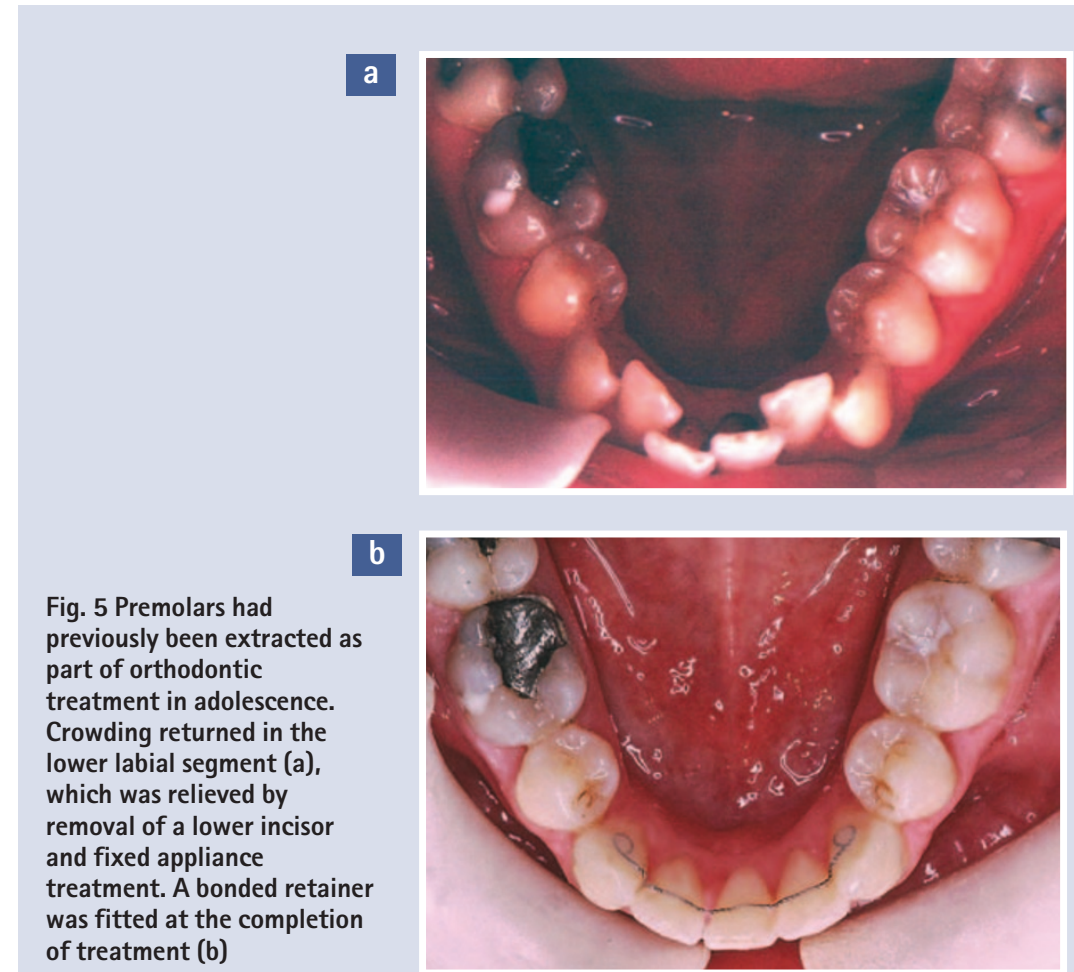

b

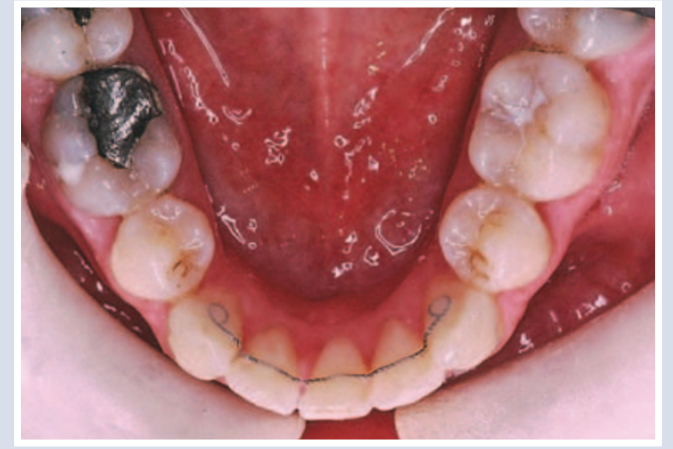
of treatment (b)
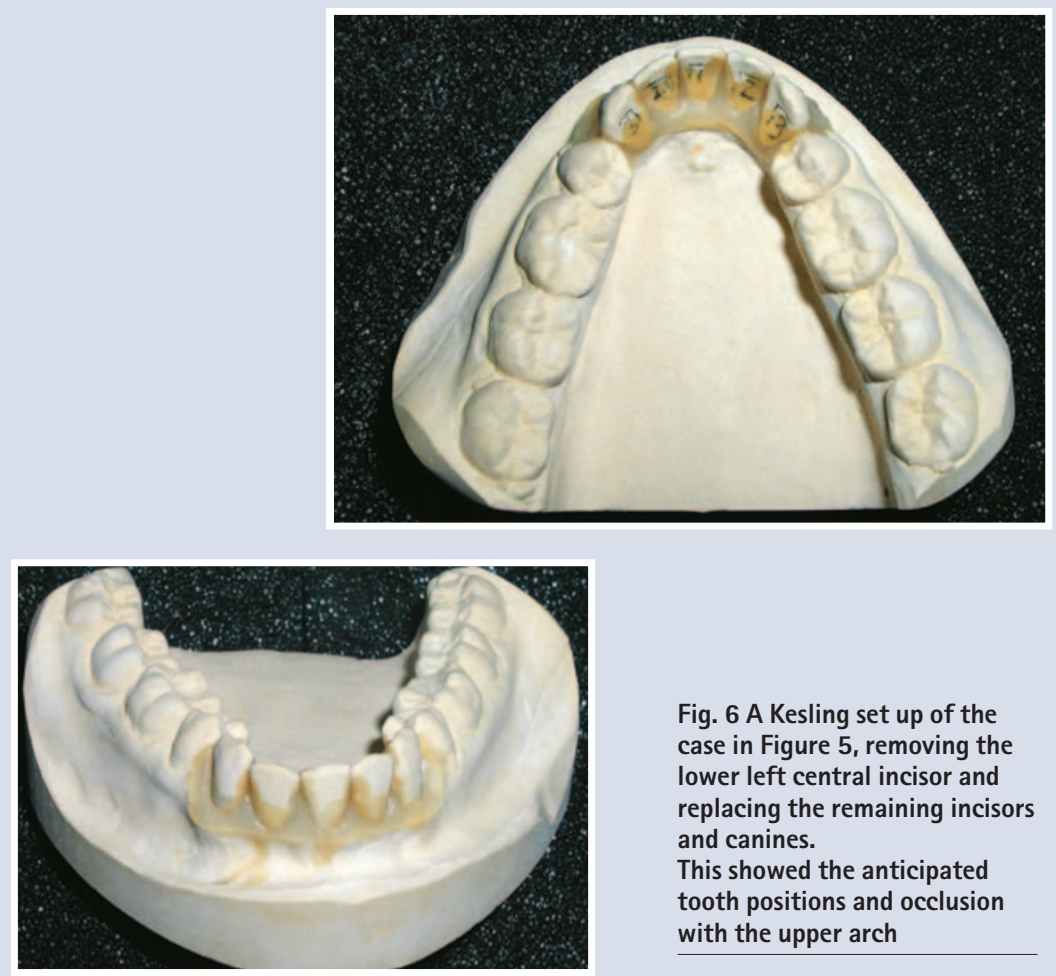

Fig. 6 A Kesling set up of the case in Figure 5, removing the lower left central incisor and replacing the remaining incisors and canines.

This showed the anticipated tooth positions and occlusion with the upper arch

Fig. 7 A lower premolar has been transplanted to replace the upper left central incisor which had a poor prognosis

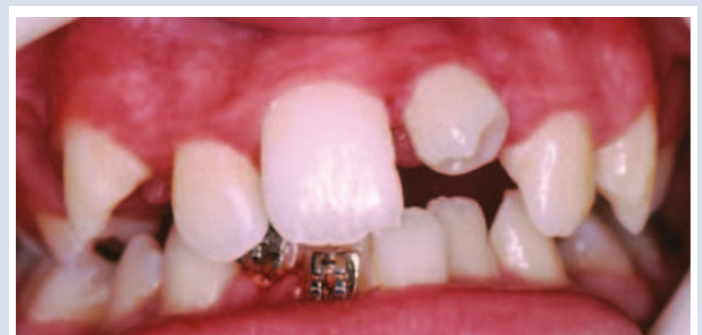




\section{PRACTICE}

a

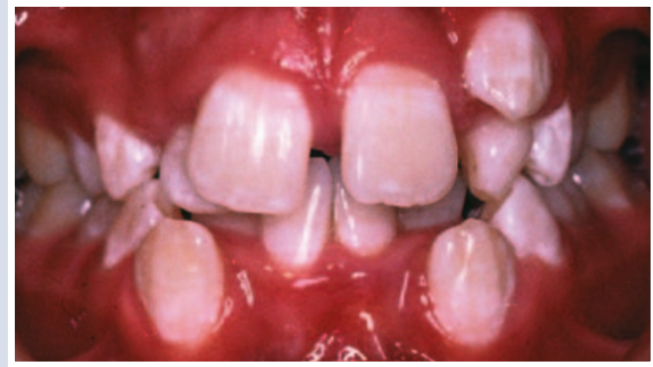

c

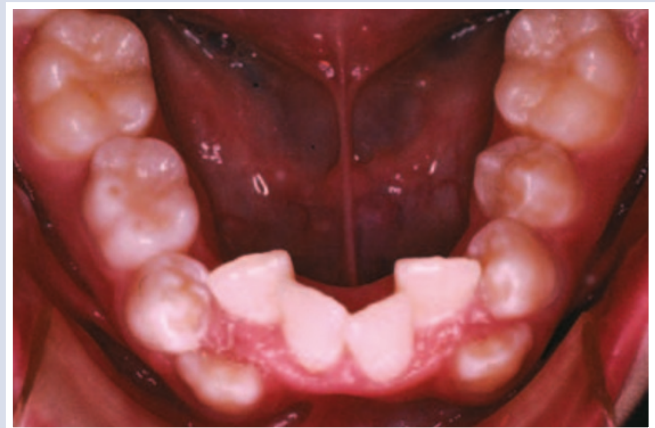

e

Fig. 8 A severely crowded case, where unusually, four canines were extracted. The resulting occlusion gave acceptable contacts between first premolar and lateral incisors and improved the arch form.

(a-c) Pre treatment, (d-f) Post treatment b

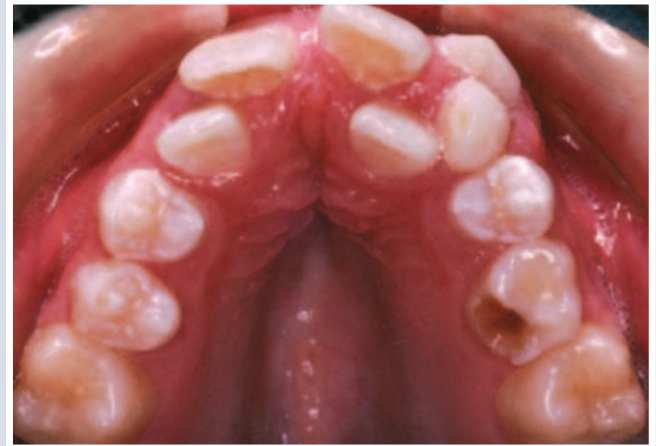

d

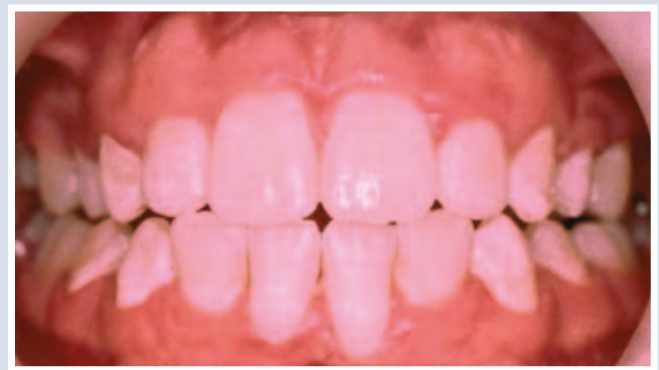

f

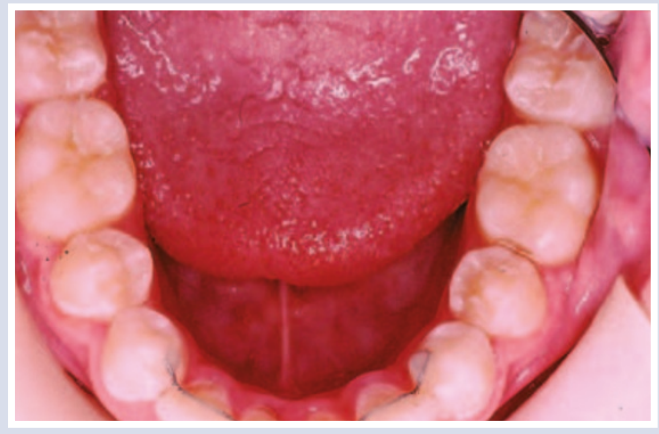

canines are mesially angulated, loss of first premolars may produce spontaneous improvement in the alignment of the canines (Fig. 9). Any excess extraction spaces may close with time, although a study by Berg et al., showed space closure to be greatest in the first 6 months following extraction. ${ }^{18}$ In carefully selected cases reasonable alignment can sometimes be achieved. However cases amenable to this type of treatment are rare and fixed appliances especially when second premolars have been extracted invariably produce better results.

Second premolars are the third most commonly developmentally absent teeth after third molars and upper lateral incisors. ${ }^{19}$ Where deciduous molars are retained beyond their normal exfoliation dates, a radiograph should be taken to confirm the presence and position of the permanent successor. In uncrowded arches deciduous molars with good roots are often retained, as space closure in these cases can be difficult (Fig. 10).

Second premolars can become impacted either due to early loss of deciduous molars or severe crowding. Ectopic second premolars usu- ally erupt lingually or palatally and should be considered for extraction if they are completely excluded from the arch (Fig. 11).

\section{First molars}

First permanent molars are often the first permanent teeth to erupt into the mouth. Their deep fissure morphology predisposes them to caries and poor tooth brushing combined with a high sugar intake, may result in gross caries. Heavily restored or decayed first molars should be considered for removal over other non-carious teeth (Fig. 12). First molars extraction requires careful planning. Their position in the arch means that whilst relief of premolar crowding is achieved the space created is far from the site of any incisor crowding or overjet reduction. The timing of the loss of first molars is also an important consideration.

Maxillary second molars have a curvilinear eruptive path with mesial and vertical components. The lower second molar has a more vertical path, but it has to move more horizontally in favourable spontaneous molar correction. This is one of the reasons why the spontaneous tooth 
a

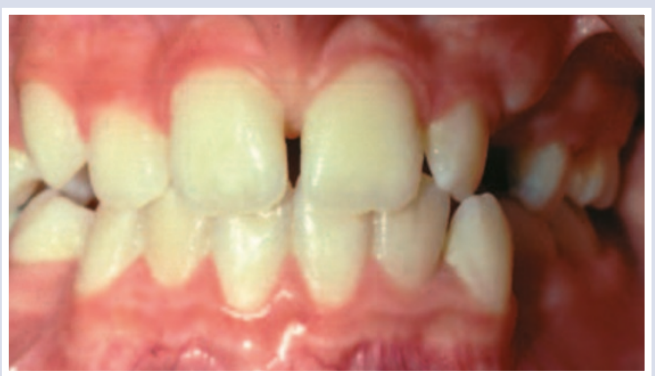

c

Fig. 9 A Class I case with mild lower labial segment crowding in the late mixed dentition (a) \& (b). All four first premolars were extracted and the occlusion allowed to align spontaneously. (c) \& (d)

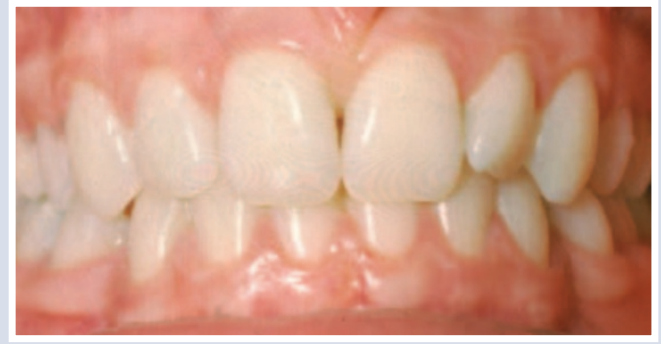

b

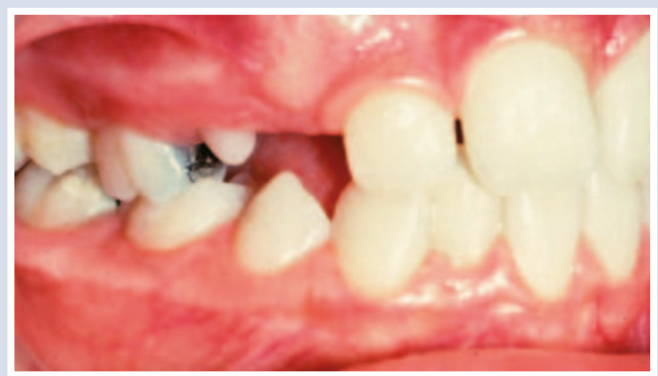

d

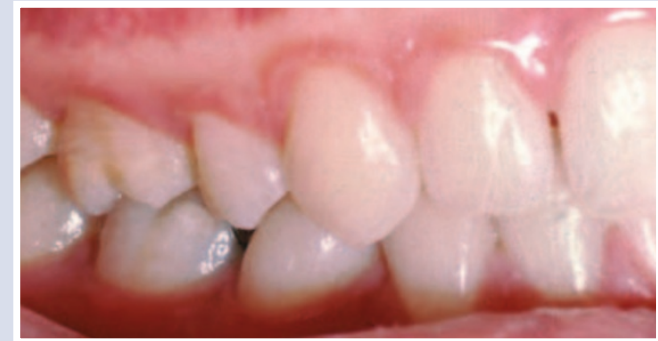

second premolar. Spontaneous relief of mild crowding in the labial segments may be seen. In the lower arch, spontaneous closure is less likely, but mesial migration of the second molar is also optimal at this stage and may resulting in minimal space between the second molar and second premolar (Fig. 12).

In the permanent dentition the effect of loss of a first molar can be difficult to predict after the second molar has erupted. Fixed appliances are invariably needed at this stage to align the teeth and achieve space closure with parallel roots. ${ }^{20}$ The effects are more of a problem in the lower arch, where the second molar tips mesially and rolls lingually forming a very poor contact with the second premolar or may leave excess space. Little spontaneous relief of anterior crowding is seen. The upper first molar if retained can over-erupt, further increasing the tipping and rolling of the lower second molar. In addition mesial movement of the lower

Fig. 10 A hypodontia case pre-treatment showing good quality deciduous molars which were retained as part of the treatment plan. Mesiodistal reduction or 'slenderising' can be used to maximise arch co-ordination, especially where deciduous molars are only retained in one arch

movement is less favourable in the lower arch. Three periods of development can be considered when looking at the effects of loss of first molars.

Maximal space closure by mesial migration of the second molar occurs in the mixed dentition. At this stage the second molars are unerupted and their root furcation is just calcifying. The best results occur in the upper arch where the second molar will usually erupt mesially and make contact with the upper

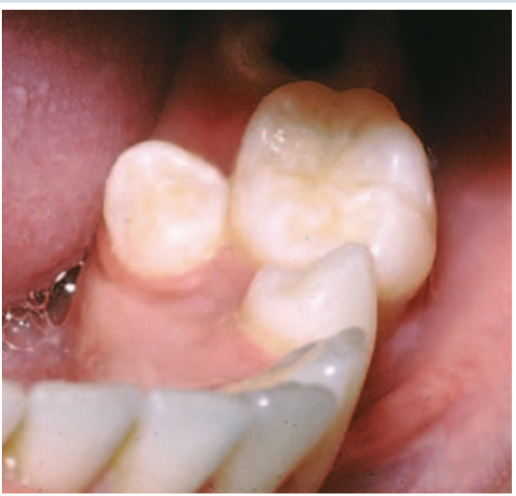

Fig. 11 Localised crowding often manifests in the lower buccal segments by lingual eruption of the second premolar 
a

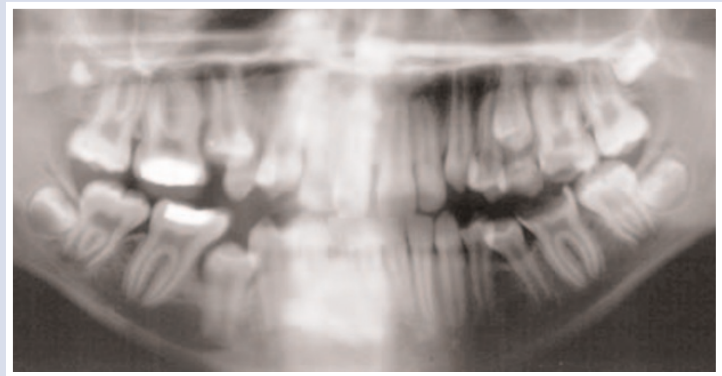

b

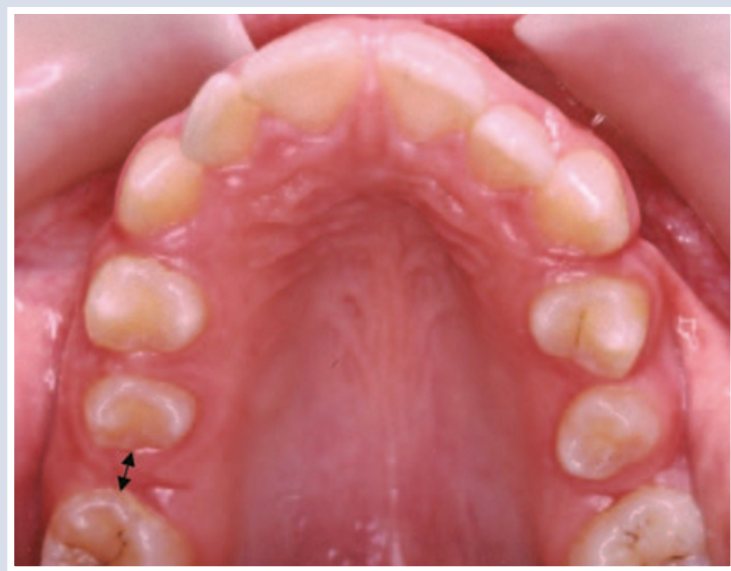

Fig. 12 Four first molars were extracted just after the optimal time, prior to fixed appliance treatment. The orthopantomogram (a) shows gross caries in the left first molars and heavy restorations in the right first molars. Notice the discrepancy in space available in the two arches. In the upper arch the second molars have erupted in close proximity to the second premolars due to their mesial eruptive path (b). In the lower arch there is considerably more space remaining from the vertical eruptive path of the second molars (c)

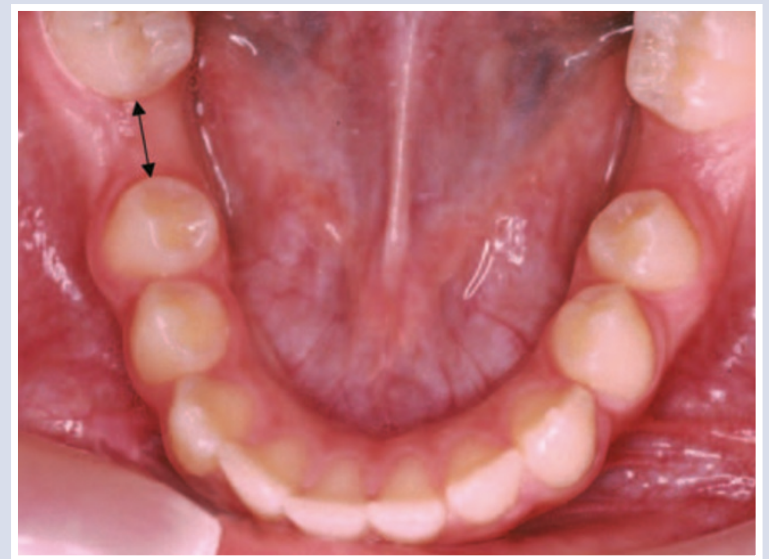

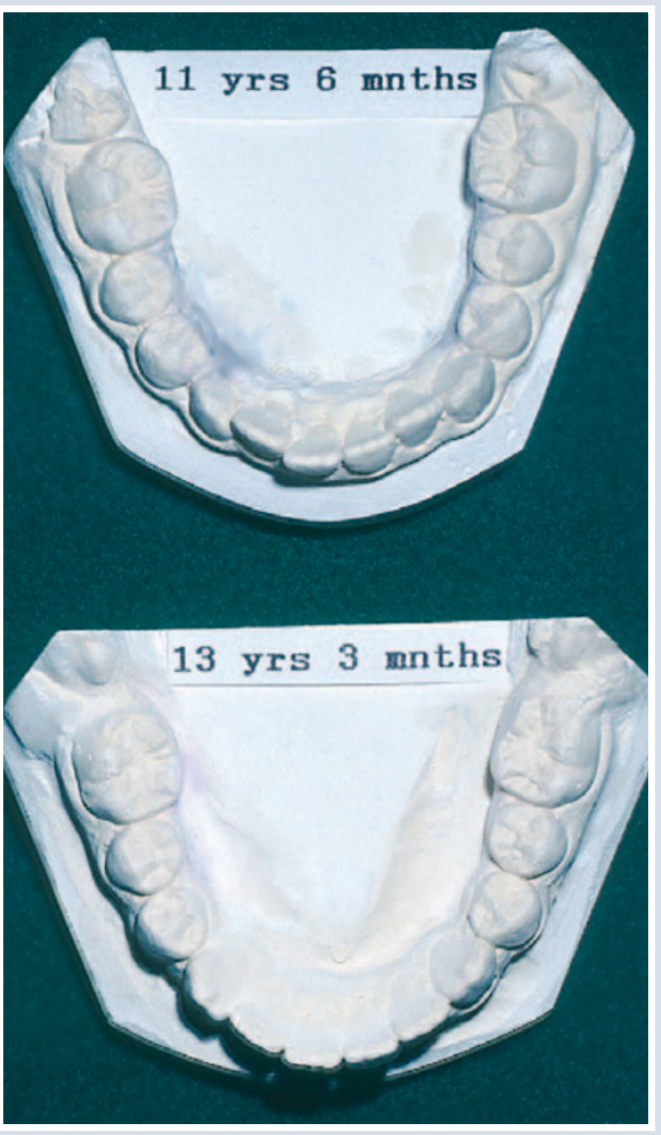

Fig. 13 Extraction of second molars allowed spontaneous relief of anterior crowding, with early eruption of the third molars molar may be prevented. The upper second molar shows less tipping and rolling than its lower counterpart, but does not align to the extent seen in the mixed dentition. In adult patients the drifting of both upper and lower second molars is less marked, and the relief of crowding less reliable. In young patients, radiographs should be checked to ensure that the developing lower second premolar is contained by the roots of the primary molar. If not, then substantial drifting of the second premolar can take place including impaction into the mesial surface of the second molar.

In general terms if a lower first molar is to be extracted, the upper molar on the same side should also be extracted (compensating extraction). This prevents unwanted overeruption of the upper first molar and the upper second molar will usually erupt into a good position. However, if an upper first molar is to be extracted, the lower counterpart is usually left in situ. This is because the lower second molar behaves unpredictably and rarely achieves good spontaneous alignment. An additional factor is that lower molars over erupt less than upper molars and will not interfere with the generally good progress made by upper second molars. If the case has no crowding, then balancing extractions should not be considered (removal of a tooth on the opposite side of the same arch). Children presenting with carious first molars often show signs of disease in all of them. If the timing is correct and the malocclusion justifies treatment, all four first molars 
should be removed to allow second molars to erupt efficiently and reduce subsequent treatment times.

\section{Second molars}

Thomas et al. ${ }^{21}$ provided a succinct summary on the role of loss of second molars in orthodontic treatment. They state that all other teeth should be present with the third molars of normal size, shape and in a good position to erupt. Mild lower labial segment crowding may be effectively treated by loss of second molars, however they should not be considered in the treatment of moderate or severe crowding. Second molar loss may be undertaken under the following circumstances:

- To facilitate the eruption of the third molars obviating the need for surgical removal at a later stage.

- To allow relief of premolar crowding (especially where second premolars are impacted)

- May prevent crowding in a well-aligned lower arch (Fig. 13).

- Distal movement in the upper arch is more reliable and more stable.

However, the potential disadvantages of second molar extraction are:

- Eruption of third molars especially in the lower arch is unpredictable. About 30\% of these teeth require uprighting.

- The teeth are remote from the site of crowding making alignment unpredictable.

Where second molars are considered for extraction, the timing is important. Satisfactory third molar alignment is less likely if the second molars are extracted after the third molar roots are more than one third formed.

\section{Third molars}

Whilst extraction of wisdom teeth for orthodontic purposes is rare, these teeth should be included in the treatment planning. The incidence of impaction of third molars varies widely in the literature. ${ }^{22}$ Posterior crowding, especially in the lower arch, may increase the risk of developing impaction. Extraction of teeth towards the front of the mouth has little effect on posterior crowding, whilst extractions towards the back improve the chances of acceptable third molars eruption. The greatest benefit occurs when second molars are removed, although eruption patterns are unpredictable. Richardson et al. ${ }^{23}$ suggest that up to $90 \%$ of third molars erupt into satisfactory positions following second molar removal, but this depends on the degree of posterior crowding and stage of root development of third molars at time of extraction. It also assumes a fairly broad minded view of what is a 'satisfactory' position.

Third molars have in the past been implicated in the aetiology of late lower incisor crowding. ${ }^{23}$ However, more recent research shows that their presence is only one of the factors involved and their influence appears to be negligible. Therefore, third molars should not be removed to relieve or prevent late lower incisor crowding. ${ }^{24}$ This forms part of the National Clinical Guidelines on the management of patients with impacted third molars. ${ }^{22}$

\section{CONCLUSIONS}

Many factors influence the choice of teeth for extraction and careful treatment planning in conjunction with good patient co-operation, appliance selection and management of the treatment are essential if an acceptable, aesthetic and functional occlusion is to be achieved.

1. Angle E H. Treatment of malocclusion of the teeth and fractures of the maxillae, Angle's system. Ed 6, 1900 S.S. Philadelphia: White Dental Manufacturing Co.

2. Wolff J. Das Gesetz der Transformation der Knochen. Berlin: Hirschwald, 1892.

3. Case CS. The question of extraction in orthdontia. Am Orthod 1964; 50: 658-691.

4. Tweed C. Clinical Orthodontics. 1966, St Louis: Mosby.

5. Begg P R. Stone age man's dentition. Am J Orthod 1954; 40: 298-312.

6. Little R M, Riedel R A, Stein A. Mandibular arch length increase during the mixed dentition: postretention evaluation of stability and relapse. Am J Orthod Dentofac Orthop 1990; 97: 393-404.

7. Betteridge M A. The effects of interdental stripping on the labial segments evaluated one year out of retention. $\mathrm{Br} J$ Orthod 1981; 8: 193-197.

8. Little R M. Stability and relapse of dental arch alignment. Br J Orthod 1990; 17: 235-241.

9. Proffit W E. Forty-year review of extraction frequency at a university orthodontic clinic. Angle Orthod 1994; 64: 407-414.

10. McCaul $L K$, Jenkins $W M$ May $E J$. The reasons for extraction of permanent teeth in Scotland: a 15-year follow-up study. BrDent J 2001; 190: 658-662.

11. Kirschen R H, O'Higgins E A, Lee R T. The Royal London Space Planning: an integration of space analysis and treatment planning: Part II: The effect of other treatment procedures on space. Am J Orthod Dentofacial Orthop 2000; 118: 448-461.

12. Khurana M, Martin M V. Orthodontics and infective endocarditis. Br J Orthod 1999; 26: 295-298.

13. Ericson $\mathrm{S}, \mathrm{Kurol}$ J. Early treatment of palatally erupting maxillary canines by extraction of the primary canines. Eur $J$ Orthod 1988; 10: 283-295.

14. Bradbury A J. The influence of orthodontic extractions on the caries indices in schoolchildren in the United Kingdom. Comm Dent Health 1985; 2: 75-82.

15. Canut J A. Mandibular incisor extraction: indications and long-term evaluation. Eur J Orthod 1986; 18: 485-489.

16. Graber T M. New horizons in case analysis: clinical cephalometrics. Am J Orthod 1956; 53: 439-454.

17. Tuverson D L. Anterior interocclusal relations. Part II. Am J Orthod 1980; 78: 371-393.

18. Berg R, Gebauer U. Spontaneous changes in the mandibular arch following first premolar extractions. Eur J Orthod 1982 4: 93-98.

19. Vastardis $H$. The genetics of human tooth agenesis: new discoveries for understanding dental anomalies. Am J Orthod Dentofac Orthop 2000; 117: 650-656.

20. Sandler PJ, Atkinson R, Murray A M. For four sixes. Am J Orthod Dentofac Orthop 2000; 117: 418-434.

21. Thomas P, Sandy J R. Should second molars be extracted? Dent Update 1995; 22: 150-156.

22. National Clinical Guidelines. The management of patients with impacted third molar (syn. Wisdom) teeth. Royal College of Surgeons of England 1997.

23. Richardson M E, Richardson A. The effect of extraction of four second permanent molars on the incisor overbite. Eur $\rfloor$ Orthod 1993; 15: 291-296.

24. Schwarze CW. The influence of third molar germectomy - a comparative long term study. Abstract of Third International Congress, London 1973; 551-562. 